\title{
Cell Migration Driven by Cooperative Substrate Deformation Patterns
}

\author{
Thomas E. Angelini, ${ }^{1}$ Edouard Hannezo, ${ }^{2}$ Xavier Trepat, ${ }^{3}$ Jeffrey J. Fredberg, ${ }^{4}$ and David A. Weitz ${ }^{1, *}$ \\ ${ }^{1}$ School of Engineering and Applied Sciences, Harvard University, Cambridge, Massachusetts 02138, USA \\ ${ }^{2}$ Département de Physique, Ecole Normale Supérieure, 75005 Paris, France \\ ${ }^{3}$ Institut de Bioenginyeria de Catalunya, Universitat de Barcelona, and Ciber Enfermedades Respiratorias, 08036 Spain \\ ${ }^{4}$ Program in Molecular and Integrative Physiological Sciences, School of Public Health, Harvard University, \\ Boston, Massachusetts 02115, USA \\ (Received 8 October 2009; published 23 April 2010)
}

\begin{abstract}
Most eukaryotic cells sense and respond to the mechanical properties of their surroundings. This can strongly influence their collective behavior in embryonic development, tissue function, and wound healing. We use a deformable substrate to measure collective behavior in cell motion due to substrate mediated cell-cell interactions. We quantify spatial and temporal correlations in migration velocity and substrate deformation, and show that cooperative cell-driven patterns of substrate deformation mediate long-distance mechanical coupling between cells and control collective cell migration.
\end{abstract}

The mechanical properties of the extracellular environment strongly influence the very elasticity of cells [1]. Moreover, cell motion, shape, and adhesion are strongly influenced by the material properties and mechanical deformations of the extracellular environment [2-4]. For example, cells spread to a maximum area on surfaces of optimal stiffness [5], and substrate elasticity can control the rate of cell migration [2]. Tracks of cell migration are persistent random walks on both soft and rigid surfaces [6,7], yet on soft surfaces, externally applied substrate deformations can alter this motion, biasing migration toward or away from a deformation source [2]. While a great deal is understood about the properties and motions of single cells, it is the behavior of collections of cells that is most important to tissue development, repair, and disease [8,9]. However, little is known about collective cell behavior and less is known about the relationship between single cell motion and collective migration. Pairs of cells in close proximity mutually alter their migration by mechanically coupling through a shared substrate [10]. Cell interactions within collections of cells are not understood, nor is it known whether pairwise interactions scale up to the multicellular level to control the collective behavior that is essential to tissues. Thus, careful investigation of multicellular interactions is essential to elucidate how collective motion can arise from cell-cell interactions.

Here, we investigate collective motion of cells in a confluent layer of epithelial cells on a deformable substrate. We find large-scale spatial patterns in the migration-velocity field of the cells, as well as in the substrate-deformation field [11]. These patterns are similar in size, growing with increasing cell density. Furthermore, the temporal evolution of the migration-velocity field follows that of the deformation field. Similar migration patterns are seen for cells growing on glass, but the patterns shrink with increasing cell density. These results show that substrate mediated cell interactions are cooperative and act over great distances, generating large-scale deformation patterns that guide collective cell migration.

A confluent island of Madin-Darby canine kidney (MDCK) cells is grown on a flat, $100 \mu \mathrm{m}$ thick polyacrylamide (PA) gel functionalized with Collagen I; the gel is homogeneous and isotropic with a Poisson's ratio of 0.5 and a shear modulus that matches the cell stiffness of $420 \mathrm{~Pa}[4,12]$. We embed beads in the PA, which is gently centrifuged during gelation to insure that the beads lie in a single plane, just below the surface. We image the beads from below to monitor substrate deformations [12,13]. Approximately 5000 cells are seeded in a drop at the center of the gel, and after the cells have adhered to the surface, the dish is filled with media and transferred to an environmentally controlled chamber on a microscope, maintained at $37{ }^{\circ} \mathrm{C}$ and $5 \% \mathrm{CO}_{2}$. We study collective behavior of the cell layer, measuring the density, migration, and substrate deformations. The cell area and migration patterns change very slowly compared to the one-minute frame rate, so data are binned in units of 200 frames (Fig. 1).

As cells migrate, they deform the PA substrate. We generate substrate-displacement fields with the method employed in traction force microscopy: we compare each image to one of the undeformed gel, determined after cells are removed by trypsin $[12,13]$. The image is divided into 1024 regions of size $16 \times 16$ pixel $^{2}$, and images of the deformed gel are cross correlated with those of the relaxed gel in each region. The resultant deformation vectors provide a deformation field for the whole image; this analysis is similar to that used in particle image velocimetry (PIV). The deformation fields exhibit coherent large-scale patterns that span many cell lengths [Figs. 2(a) and 2(b)]; by contrast, the deformation field around an isolated single cell on PA gel extends only just past the cell periphery $[12,14,15]$. This suggests that within the confluent layer, single-cell contractions are balanced by forces transmitted from cell to cell, reducing the substrate stretching. These 

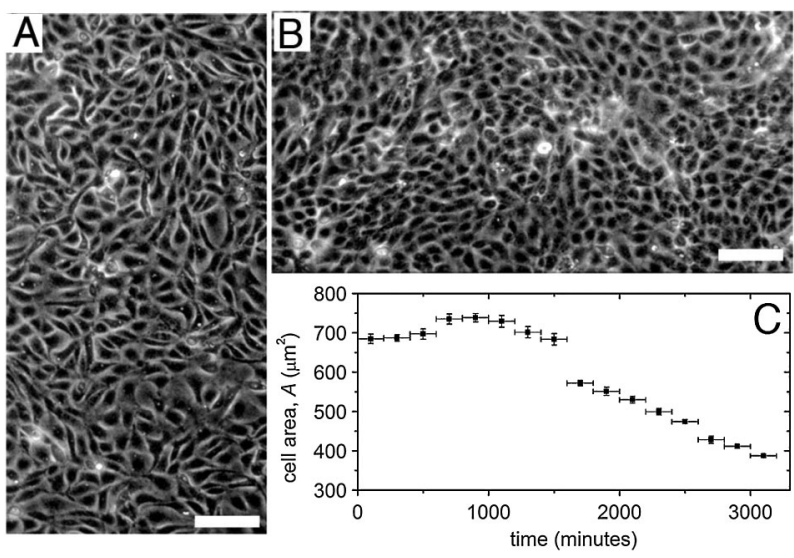

FIG. 1. Cell density is low and cell area is large at early times (a), and cells compact over time (b). Initially, cell area increases slightly, as island expansion just exceeds changing cell density due to proliferation. After 900 minutes, average cell area decreases and cell density steadily increases (c). Scalebar $=$ $100 \mu \mathrm{m}$.

patterns can only be detected by comparing the deformed to the relaxed substrate; analysis of frame-to-frame motion shows only local fluctuations, similar to those seen for isolated single cells.

A characteristic length scale is apparent in these multicellular patterns. We quantify this length scale with a spatial correlation function that averages the scalar product
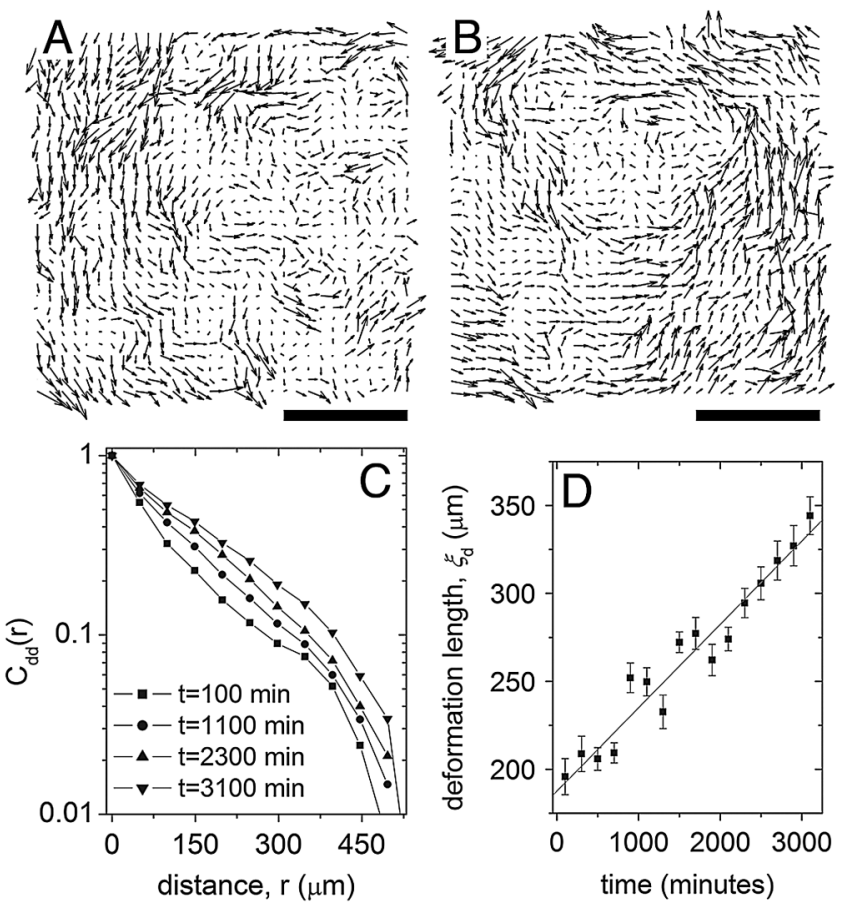

FIG. 2. Substrate-displacement fields at low cell densities (a) and high cell densities (b) contain long-distance, complex patterns. Scalebar $=248 \mu \mathrm{m}$. The spatial autocorrelation function of displacement vectors, $C_{\mathrm{dd}}(r)$, decays exponentially (c), with a characteristic deformation correlation length, $\xi_{d}$, that grows over time as cell density increases (d). of all vector pairs separated by a distance, $R$,

$$
C_{\mathbf{x} \mathbf{x}}(R)=\left\langle\frac{\sum_{j} \delta \mathbf{x}\left(\mathbf{r}_{j}\right) \cdot \delta \mathbf{x}\left(\mathbf{r}_{j}+\mathbf{R}\right)}{\sum_{j} \delta \mathbf{x}\left(\mathbf{r}_{j}\right) \cdot \delta \mathbf{x}\left(\mathbf{r}_{j}\right)}\right\rangle_{t, \varphi}
$$

where the angle brackets signify an average over all directions and time, and the sums are taken over all positions $\mathbf{r}_{j}$. For the displacement vector correlation function, $C_{\mathbf{d d}}(R)$, we make the substitution $\mathbf{x}(\mathbf{r})=\mathbf{d}(\mathbf{r})$. Rather than autocorrelating the displacement fields, $\mathbf{d}(\mathbf{r})$, we first subtract the mean displacement vector of each frame, $\overline{\mathbf{d}}$, and autocorrelate the displacement fluctuation field, $\delta \mathbf{d}(\mathbf{r})=$ $\mathbf{d}(\mathbf{r})-\overline{\mathbf{d}}$. This field has a zero mean, reducing edge effects and asymmetries in the correlation function at the largest length scales. This correlation function is similar in spirit to that used for quantifying the size of swirls in sedimenting colloidal particles [16]. For each of our measurements, $C_{\mathbf{d d}}(R)$ decays exponentially over hundreds of microns and can be well fit within this range using the function $C_{\mathbf{d d}}(R)=\exp \left(-R / R_{0}\right)$, [Fig. 2(c)]. Thus, a displacement vector at any point is randomly correlated with displacement vectors at distances larger than $R_{0}$; this defines the domain size of correlated deformations, $\xi_{d}=$ $2 R_{0}$. We find that $\xi_{d}$ is much larger than the size of a cell and varies systematically with cell density, from around $200 \mu \mathrm{m}$ at low densities to $350 \mu \mathrm{m}$ at high densities, shown in Fig. 2(d).

As cells move over these deformation fields, they must migrate cooperatively since there is no free space. To quantify collective cell migration that can be directly compared to the substrate-displacement fields at the same locations over long times, we compute velocity fields instead of tracking individual cells. We employ a PIV-like analysis that measures a displacement field per unit time for successive phase-contrast images of the cell layer. As with the substrate-displacement fields, large-scale patterns are evident in the velocity fields [Figs. 3(a) and 3(b)] [11]. The spatial extent of these patterns is a measure of the domain size of collective cell motion within the layer. To quantify the size of these coordinated domains, we use Eq. (1), substituting $\mathbf{x}(\mathbf{r})=\mathbf{v}(\mathbf{r})$. We again use the fluctuation field, $\delta \mathbf{v}(\mathbf{r})=\mathbf{v}(\mathbf{r})-\overline{\mathbf{v}}$, subtracting the average velocity vector, $\overline{\mathbf{v}}$, which is always much smaller than the fluctuations, negligibly altering the overall vector field. In contrast to $C_{\mathbf{d d}}(R)$, which decays monotonically, $C_{\mathbf{v v}}(R)$ shows a clear negative minimum [Fig. 3(c)]. The difference between $C_{\mathbf{d d}}(R)$ and $C_{\mathbf{v v}}(R)$ arises because the velocity fluctuation patterns are swirls. The scalar product in the correlation functions give negative numbers for all pairs of opposing vectors, and antiparallel velocity vectors on opposite sides of swirl patterns produce an average negative correlation value. The correlation function invariably exhibits a well-defined minimum corresponding to the size of the swirls, and its position enables the determination of a migration correlation length, or swirl size, $\xi_{s}$ [16]. At early times, when density is low, $\xi_{s}$ is small; at later times, when density is high, $\xi_{s}$ becomes large [Fig. 3(d)]. 


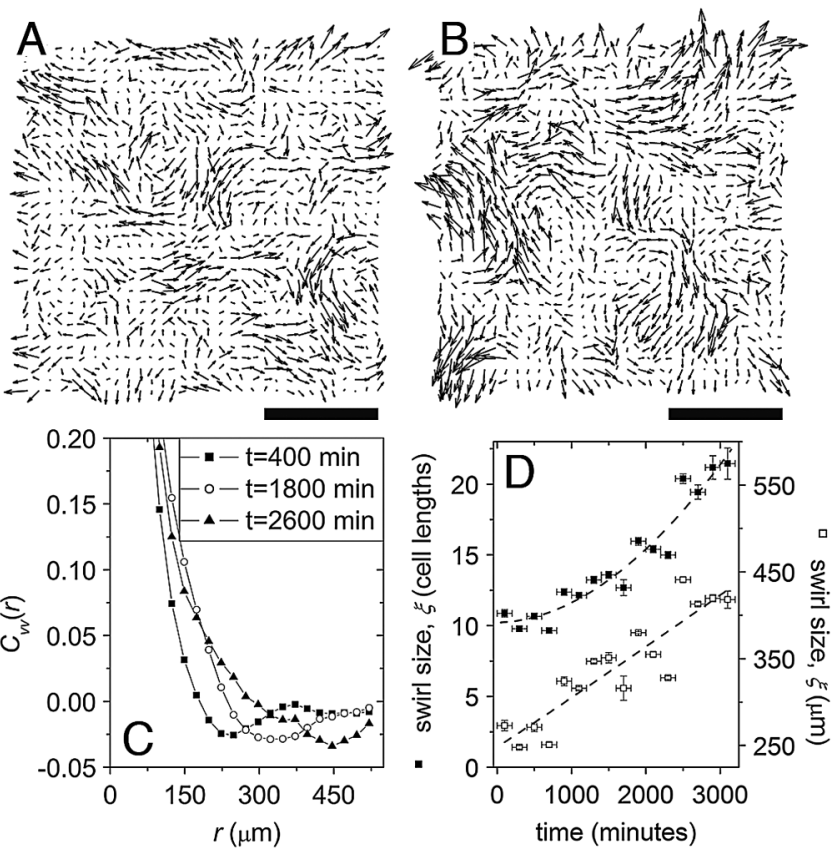

FIG. 3. Velocity fluctuation fields of cell migration show largescale swirl patterns resembling the underlying substrate (a), low cell density; (b), high cell density; Scalebar $=248 \mu \mathrm{m}$. The spatial autocorrelation function of velocity fluctuation vectors, $C_{\mathrm{vv}}(r)$, decays over short distances and shows a clear negative minimum at larger distances (c). The position of this minimum defines a swirl correlation length, $\xi_{s}$, which grows over time as cell density increases (d).

At lower densities, cell shapes fluctuate dramatically, whereas at higher densities, cell shapes change little and remain nearly round. Thus, smaller rounder cells coordinate motion in larger numbers of cells and over greater distances than do the larger more dynamic cells.

The deformation fields observed here are a type of surface pattern, but are directly generated by the cells. Patterned surfaces can control cell migration $[17,18]$, and our data allow us to determine the role of multicellular deformation patterns in guiding cell motion. We find a direct, linear correlation between $\xi_{d}$ and $\xi_{s}$ [Fig. 4(a)]. To investigate the influence of substrate deformability on collective migration, we measure cell behavior on a rigid glass surface. We find a dramatic difference: on PA gels, $\xi_{s}$ is inversely proportional to cell projected area [Fig. 4(b)]. By contrast, collective swirl patterns are still observed within the cell layer on glass, and cells proliferate at the same rate as on PA gels, but the trend is opposite to the case of PA gels; $\xi_{s}$ grows with increasing projected cell area [Fig. 4(c)]. Thus, the deformability of the substrate changes the number of cells that move collectively at a given density. On rigid substrates, cells at high density move in small domains; on soft substrates, cells at high density move in large domains.

If large-scale substrate deformations guide collective cell motion in a causal manner, then at each location, changes in substrate deformation should precede changes
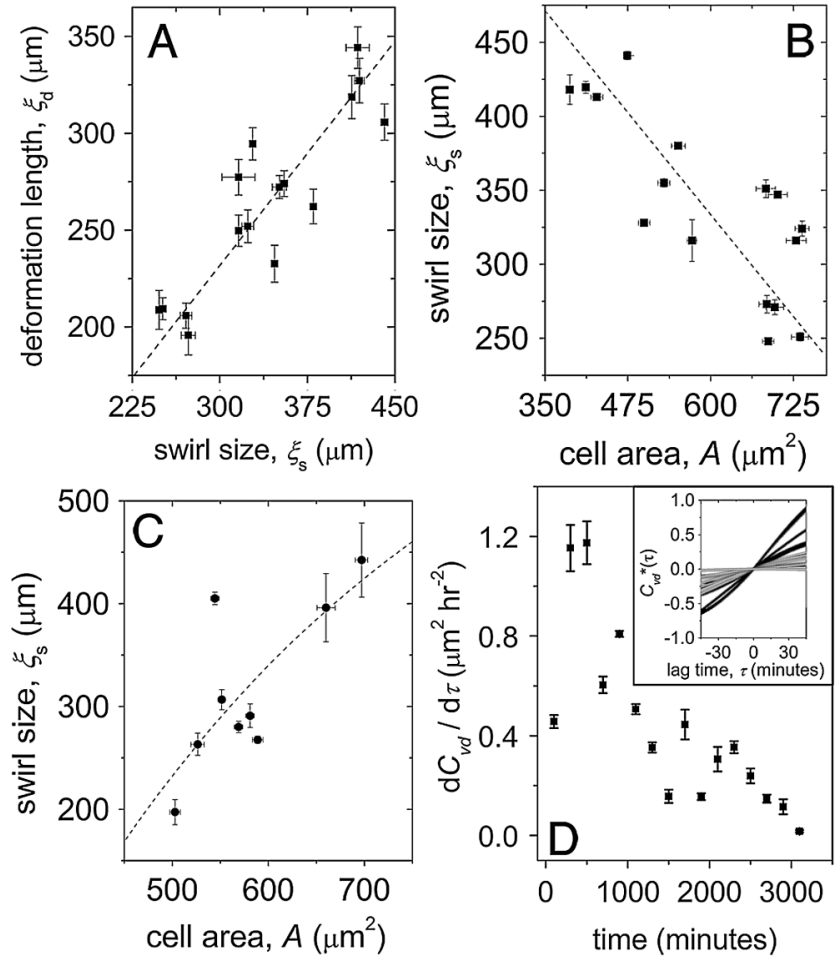

FIG. 4. $\xi_{d}$ increases with the increasing $\xi_{s}$ (a). For cells on PA substrates, $\xi_{s}$ increases with increasing density (b); the opposite trend is seen on glass substrates (c). The temporal crosscorrelation function of velocity fluctuations and substrate displacements, $C_{\mathbf{d v}}(\tau)$, shows that velocity fluctuations lag substrate deformations in time [(d), inset]. The rate of these reorientations decreases throughout the experiment as cell density increases. In the inset, we shift the cross-correlation functions, $C_{\mathbf{d v}}(\tau)^{*}=$ $C_{\mathbf{d v}}(\tau)-C_{\mathbf{d v}}(0)$, preserving the trends in $C_{\mathbf{d v}}(\tau)$, shifting all data to fit in one plot.

in cell migration. We investigate this relationship with a temporal cross-correlation function that compares a displacement vector, $\delta \mathbf{d}$, and a velocity vector, $\delta \mathbf{v}$, at a given location; this quantifies the extent to which $\delta \mathbf{d}$ is correlated with $\delta \mathbf{v}$ at another time, $\tau$,

$$
C_{\mathbf{d v}}(\tau)=\left\langle\sum_{j} \delta \mathbf{d}\left(t_{j}\right) \cdot \delta \mathbf{v}\left(t_{j}+\tau\right)\right\rangle_{\mathbf{R}}
$$

where brackets signify an average over all positions in the vector fields, and the sum is taken over all times, $t_{j}$. From these cross-correlation functions, we find that velocity fluctuations lag substrate displacements in time. Given a substrate displacement, $\delta \mathbf{d}$, at any time, velocity fluctuations at future times are always more correlated with $\delta \mathbf{d}$ than at previous times [Fig. 4(d) (inset)]. This unambiguously shows that migration-velocity fluctuations follow substrate-displacement fluctuations. To track how migration fluctuations change over time in response to substrate displacements, we calculate the average slope of the correlation function, $\left\langle\partial C_{\mathbf{d v}}(\tau) / \partial \tau\right\rangle_{\tau}$, for $-10 \mathrm{~min} \leq \tau \leq$ $10 \mathrm{~min}$. At early times, at low cell densities, migration quickly responds to substrate displacements; at later times, 
at high cell densities, the cell layer responds more slowly to substrate displacements [Fig. 4(d)]. In all cases, $\left\langle\partial C_{\mathbf{d v}}(\tau) / \partial \tau\right\rangle_{\tau}$ is positive, indicating that velocity fluctuations always lag substrate displacements.

This study establishes that multicellular mechanical cooperativity guides collective cell migration. The growth of $\xi_{s}$ with density could arise from a combination of shortrange interactions and high cell density, as observed in driven particle systems [19-21], as well as in bacterial and eukaryotic cell systems [22,23]. However, our observation that $\xi_{s}$ decreases with increasing density on glass, in complete contrast to the behavior seen on the deformable substrate, suggests that a different kind of cell-cell or cellsubstrate interaction must control MDCK behavior. On soft surfaces, not only local interactions are important; instead, the long-distance substrate-deformation fields that emerge from these nearest-neighbor interactions control collective motion. Moreover, mechanical communication through the extracellular environment must strongly influence any chemical signals involved in collective migration, such as morphogens, since correlated motion changes dramatically when only the substrate rigidity is changed; thus, any understanding of chemical signaling in collective motion in tissues must include coupling between chemical signals, ECM deformations, and spatial correlations in cell motion [24-26]. However, we cannot rule out that this difference arises because secreted chemical signals cannot diffuse between cells on their apical side through the glass.

Collective MDCK motion has been seen on fibrous substrates, in which the cell layer reorganizes collagenfiber bundles in the extracellular matrix and migrates through contact guidance [27]. Our results show that cells can organize mechanically guided motion in the absence of oriented fibers. One surprising feature of the type of mechanically guided motion seen here is that a large group of cells can collectively deform a substrate in the same direction. This requires cells to balance forces not merely by traction, since traction forces for single cells in isolation must be balanced locally. In recent experiments on a similar system, the whole cell island is shown to be a contractile unit, deforming the substrate toward the center, where tensile stresses that balance the collective traction forces are transmitted through cell-cell junctions [28]. Our results show that this transmission of force across many cells generates large-scale deformation patterns that can guide their motion, and similar experiments in which cellcell junctions such as Cadherins are blocked could further elucidate the underlying mechanism. In contrast to other classes of mechanically guided motion, like contact guidance on fibrous surfaces or multicellular "purse-strings" that guide embryonic dorsal closure and wound healing $[9,29]$, the mechanical guide of motion observed here does not depend on the reconstruction of cellular or extracellular material; it merely requires their deformation. Thus, this guiding mechanism may be important to collective motions in tissues, and our results with MDCK cells will serve as a benchmark for future studies on other systems.
This work was supported by the NSF (DMR-0602684) and the Harvard MRSEC (DMR-0820484). We would like to thank Dr. James Butler for helpful conversations.

*Corresponding author: David A. Weitz, Pierce Hall, 29 Oxford Street, Cambridge, MA 02138. Phone: 617-4962842. Fax: 617-495-0426; weitz@seas.harvard.edu

[1] N. Wang et al., Am. J. Physiol., Cell Physiol. 282, C606 (2002).

[2] C. M. Lo et al., Biophys. J. 79, 144 (2000).

[3] K. Keren et al., Nature (London) 453, 475 (2008).

[4] D. E. Discher, P. Janmey, and Y.-1. Wang, Science 310, 1139 (2005).

[5] A. Engler et al., Biophys. J. 86, 617 (2004).

[6] G. A. Dunn, Agents Actions Suppl. 12, 14 (1983).

[7] R. B. Dickinson and R. T. Tranquillo, AIChE J. 39, 1995 (1993).

[8] P. Friedl, Y. Hegerfeldt, and M. Tusch, Int. J. Dev. Biol. 48, 441 (2004).

[9] P. Martin and S. M. Parkhurst, Development (Cambridge, U.K.) 131, 3021 (2004).

[10] C. A. Reinhart-King, M. Dembo, and D. A. Hammer, Biophys. J. 95, 6044 (2008).

[11] See supplementary material at http://link.aps.org/ supplemental/10.1103/PhysRevLett.104.168104 for timelapse movies of the cell layer, and the velocity and substrate displacement fields.

[12] M. Dembo and Y.-L. Wang, Biophys. J. 76, 2307 (1999).

[13] K. A. Beningo, C. M. Lo, and Y. L. Wang, in Methods in Cell-Matrix Adhesion (Academic Press, San Diego, 2002), p. 325 .

[14] J. P. Butler et al., Am. J. Physiol., Cell Physiol. 282, C595 (2002).

[15] B. Sabass et al., Biophys. J. 94, 207 (2008).

[16] P. N. Segrè, E. Herbolzheimer, and P. M. Chaikin, Phys. Rev. Lett. 79, 2574 (1997).

[17] D. S. Gray, J. Tien, and C. S. Chen, Journal of Biomedical Materials Research. Part A 66A, 605 (2003).

[18] X. Jiang et al., Proc. Natl. Acad. Sci. U.S.A. 102, 975 (2005).

[19] J. Toner and Y. Tu, Phys. Rev. E, Stat. Nonlinear Soft Matter Phys. 58, 4828 (1998).

[20] I. S. Aranson, D. Volfson, and L. S. Tsimring, Phys. Rev. E 75, 051301 (2007).

[21] J. Toner, Y. Tu, and S. Ramaswamy, Ann. Phys. (N.Y.) 318, 170 (2005).

[22] A. Sokolov et al., Phys. Rev. Lett. 98, 158102 (2007).

[23] B. Szabo et al., Phys. Rev. E, Stat. Nonlinear Soft Matter Phys. 74, 061908 (2006).

[24] R.C. Kurten et al., Am. J. Physiol. Cell Physiol. 288, C109 (2005).

[25] J. B. Wallingford et al., Curr. Biol. 11, 652 (2001).

[26] D. Dormann and C. J. Weijer, Development (Cambridge, U.K.) 128, 4535 (2001).

[27] H. Haga et al., Biophys. J. 88, 2250 (2005).

[28] X. Trepat et al., Nature Physics 5, 426 (2009).

[29] M.S. Hutson et al., Science 300, 145 (2003). 